5. Availability of Tables. The table described in the present article has been deposited in the file of Unpublished Mathematical Tables which MTAC maintains. This material may be made available on loan to interested people [2].

University of Illinois

G. BELFORD

Urbana, Illinois

and

Midwestern Universities Research Association

On leave of absence from

L. JACKSON LASLETT

Iowa State College

Ames, Iowa

J. N. SNYDER

University of Illinois

Urbana, Illinois

and

Midwestern Universities Research Association

The authors were assisted by the National Science Foundation and the Office of Naval Research.

1. E. T. Whittaker \& G. N. Watson, A Course of Modern Analysis, Cambridge Univ. Press, 1946, p. $412 \mathrm{ff}$.

2. G. BeLFord, L. JACKSON LASLETT, \& J. N. SNYDER, Table Pertaining to Solutions of a Hill Equation [MTAC, this issue, Review 59, p. 110].

\title{
Rational Formulae for the Production of a Spherically Symmetric Probability Distribution
}

Von Neumann [1] has described the following method for producing a uniform distribution on the circumference of the unit circle: Select two random numbers $x_{1}, x_{2}$ from a uniform distribution on $(-1,+1)$. Reject the pair if $x_{1}{ }^{2}+x_{2}{ }^{2} \geq 1$, and re-select until a pair is obtained satisfying $x_{1}{ }^{2}+x_{2}{ }^{2}<1$. Then, by the trigonometric double-angle identities,

$$
x=\frac{x_{1}^{2}-x_{2}^{2}}{x_{1}^{2}+x_{2}^{2}}, \quad y=\frac{2 x_{1} x_{2}}{x_{1}^{2}+x_{2}^{2}}
$$

are the co-ordinates of a point having the desired distribution.

The following is an extension of this method for points distributed on a sphere uniformly with respect to area. Select four random numbers $x_{0}, x_{1}, x_{2}, x_{3}$ from a uniform distribution on $(-1,+1)$. Reject the quadruplet if $x_{0}{ }^{2}+x_{1}{ }^{2}+x_{2}{ }^{2}+x_{3}{ }^{2} \geq 1$, and re-select until a quadruplet is obtained satisfying $x_{0}{ }^{2}+x_{1}{ }^{2}+x_{2}{ }^{2}+x_{3}{ }^{2}<1$. Then

$x=\frac{2\left(x_{1} x_{3}+x_{0} x_{2}\right)}{x_{0}{ }^{2}+x_{1}{ }^{2}+x_{2}{ }^{2}+x_{3}{ }^{2}}, \quad y=\frac{2\left(x_{2} x_{3}-x_{0} x_{1}\right)}{x_{0}{ }^{2}+x_{1}{ }^{2}+x_{2}{ }^{2}+x_{3}{ }^{2}}, \quad z=\frac{x_{0}{ }^{2}+x_{3}{ }^{2}-x_{1}{ }^{2}-x_{2}{ }^{2}}{x_{0}{ }^{2}+x_{1}{ }^{2}+x_{2}{ }^{2}+x_{3}{ }^{2}}$

are the co-ordinates of a point uniformly distributed over the surface of the unit sphere. 
A proof depends on the fact that a non-zero quaternion $q=x_{0}+x_{1} \mathbf{i}+x_{2} \mathbf{j}+x_{3} \mathbf{k}$ effects the rotation $\mathbf{\nabla} \rightarrow \mathbf{q v q}^{-1}$ of vectors $\mathbf{\nabla}$, and that conversely every rotation can be so produced by some quaternion.

Select $\mathbf{q}$ from a uniform distribution on the set $|\mathbf{q}|<1$ as above. Then $\mathbf{q} \mathbf{k q}^{-1}$ will have on the surface of the unit sphere a distribution invariant under rotations because, if $\mathbf{p}$ is any other non-zero quaternion, then

$$
\mathbf{p}\left(\mathbf{q} \mathbf{k} \mathbf{q}^{-1}\right) \mathbf{p}^{-1}=\left(\frac{\mathbf{p}}{|\mathbf{p}|} \mathbf{q}\right) \mathbf{k}\left(\frac{\mathbf{p}}{|\mathbf{p}|} \mathbf{q}\right)^{-1}
$$

but multiplication of $\mathbf{q}$ by a quaternion of unit norm is a rotation of quaternion space and hence preserves the distribution from which we selected $\mathbf{q}$.

But there exists on the surface of a sphere only one distribution invariant under all rotations, namely, the uniform distribution.

Now let $\mathbf{q} \mathbf{k q} \mathbf{q}^{-1}=x \mathbf{i}+y \mathbf{j}+z \mathbf{k}$ and we have the above formulae.

The method extends to $n$ dimensions and to more general spaces acted upon by a compact group of transformations.

\section{J. M. Cook}

Argonne National Laboratory

Lemont, Illinois

This work was performed under the auspices of the U.S. Atomic Energy Commission.

1. John von NeumanN, "Various techniques used in connection with random digits," NBS Applied Mathematics Series, No. 12, U. S. Gov. Printing Office, Washington, D. C., 1951, p. 36-38.

\section{On the Numerical Solution of Integral Equations}

For the numerical solution of linear integral equations we must of course replace all our variables, both dependent and independent, by discrete variables. In choosing the grid for the independent variables we should naturally be influenced by the storage facilities of our calculating machines. One purpose of this note is to emphasize the obvious fact that we should not be too strongly influenced by the storage facilities since we may then take longer than necessary for the job. The following technique is formulated in terms of finding the inverse of a kernel, $K(x, y)$, but a similar technique could be applied for solving a single integral equation. The idea seems too simple to be new but I have not met it before, and it may have some applications.

First choose a wide grid. This will convert our kernel into a small matrix, $M_{1}$. Invert it. Next choose a less wide grid, and hence a larger matrix, $M_{2}$. Use the result of stage 1 to construct an approximate inverse of $M_{2}$ (by smoothing the elements of the inverse of $M_{1}$ so as to obtain a matrix of the right size). This step will usually be justifiable if $K(x, y)$ is a smooth function. Starting with our approximate inverse of $M_{2}$ we may obtain a more exact inverse by means of a well known iterative procedure known to be rapidly convergent when we start with a good approximation (I. J. Good [1] and H. Hotelling [2]). We can next choose a still narrower grid and proceed as before. The whole process can be stopped when adjacent entries in our matrix are numerically close. 